\title{
FENADOCE: Uma experiência gastronômica ou um símbolo de pertencimento para os residentes?
}

\section{FENADOCE: A gastronomic experience or a symbol of belonging for residents?}

\author{
Cristiane Berselli \\ Universidade do Vale do Itajai (UNIVALI), Brasil \\ cristiane.berselli@gmail.com \\ Fabricia Durieux Zucco \\ Universidade do Vale do Itajai (UNIVALI), Brasil \\ fabriciazucco@hotmail.com \\ Edar da Silva Añaña \\ Universidade Federal de Pelotas (UFPEL), Brasil \\ edar@ufpel.edu.br
}

\begin{abstract}
Resumo
Eventos e festivais são manifestações culturais que impulsionam a economia local, atraem turistas e reforçam a identidade e os laços afetivos dos moradores. $O$ artigo avalia o relacionamento de três importantes elementos da Feira Nacional do Doce - FENADOCE: o evento, a sua organização e seus impactos; o sentimento de pertença dos moradores em relação ao festival; e a posição da mascote como marca-símbolo do evento. Os 424 questionários recolhidos de residentes da cidade de Pelotas-RS (Brasil) foram analisados através de Análise Fatorial e Modelagem de Equações Estruturais. O resultado identificou sete dimensões de análise: organização do evento; benefícios socioculturais; benefícios socioeconômicos; impactos negativos; pertencimento dos residentes; valor econômico e valor simbólico da mascote. O trabalho oferece contribuições teóricas e gerenciais na gestão de marcas de destinos ou de eventos, especialmente aquelas que se fazem representar por meio de mascotes, cuja incorporação ao ideário dos residentes nem sempre é tarefa fácil.
\end{abstract}

Palavras-chave: Evento; FENADOCE; impactos percebidos; mascote; pertencimento; residentes.

\begin{abstract}
Events and Festivals are cultural events that boost the local economy, attract tourists and reinforce the identity and affective ties of residents. The article assesses the relationship of three important elements of the Feira Nacional do Doce - FENADOCE: the event, its organization and its impacts; the residents' feeling of belonging to the festival; and the position of the mascot as a symbol of the event. Data of the 424 questionnaires collected from residents of the city of Pelotas-RS (Brazil) were analyzed through Factor Analysis and Structural Equation Modeling. The result identified seven dimensions of analysis: organization of the event; socio-cultural benefits; socioeconomic benefits; negative impacts; residents' belongingness; economic value; and symbolic value of the mascot. The work offers theoretical and managerial contributions in the fields of destination
\end{abstract}

Dos Algarves: A Multidisciplinary e-Journal, 39-2021.

ISBN 2182-5580 @ ESGHT - University of the Algarve, Portugal.

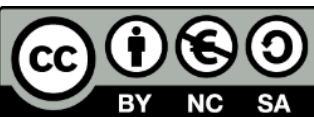

To cite this article: Berselli, C., Zucco, F. D. \& Añaña, E. S. (2021). FENADOCE: Uma experiência gastronômica ou um símbolo de pertencimento para os residentes? Dos Algarves: A Multidisciplinary e-Journal, 39, 15-36. DOI: 10.18089/DAMeJ.2021.39.2 
and/or event's brands, especially those represented by mascots, whose incorporation into the residents' ideals is not always an easy task.

Keywords: Event; FENADOCE; perceived impacts; mascot; belonging; residents.

\section{Introdução}

Tal como acontece com os produtos em geral, os destinos turísticos precisam exaltar os atributos que os diferenciam dos demais para atrair e encantar visitantes, e os eventos, quando geridos de forma integrada e coerente com os demais atrativos, podem representar um diferencial para o destino (Guerreiro, Oom do Valle \& Mendes, 2011; Getz \& Page, 2016). O turismo de eventos é um dos segmentos mais interessantes do ponto de vista financeiro, tendo em vista que os turistas que a eles acorrem "ficam mais tempo no destino e frequentemente viajam em grupo” (Kelly \& Fairley, 2018: 335). Este setor é um dos que melhor aproveita o efeito multiplicador proporcionado pela atividade turística, pois as empresas e a comunidade anfitriã são beneficiadas direta e indiretamente, antes, durante e após a realização do evento.

Além dos benefícios econômicos, os eventos e festivais ajudam a difundir a cultura local e fomentam o orgulho das comunidades, colaborando com a preservação das tradições locais e do patrimônio cultural (Gursoy, Kim \& Uysal, 2004; Stankova \& Vassenska , 2015), fortalecem a coesão, melhoram a qualidade de vida e reforçam a imagem da comunidade (Gursoy et al., 2004) e, além disso, celebram os valores, as ideologias e continuidade da identidade da comunidade (Getz, Andersson \& Carlsen, 2010). Em última análise, os eventos locais possibilitam que turistas e moradores vivenciem a cultura local na sua forma mais autêntica (Akhoondnejad, 2016).

No marketing, a identidade da marca (aquela que é pretendida pela gestão) deve ser igual à imagem percebida (Vásquez, 2007). No turismo, Pimentel, Pinho e Vieira (2006) afirmam que a marca de um destino turístico deve ser congruente com a identidade local. Ainda, a marca sustentável é aquela desenvolvida conforme os valores da comunidade, no entanto, nem sempre esses valores são adequadamente percebidos (Wheeler, Frost \& Weiler, 2011). Quando há discrepâncias entre os valores da comunidade e aqueles percebidos pelos turistas através da marca, é possível que a atividade turística seja mal vista por uns ou por outros. Ademais, os impactos percebidos do turismo estão diretamente relacionados à formação da imagem (Rola, Malheiro \& Sousa, 2018), o que requer esforços dos gestores de marca e branding para mitigarem as percepções dos impactos negativos e criarem estratégias para que os benefícios da atividade se destaquem.

Além da identidade comunicada através da marca e dos impactos positivos e negativos percebidos, propomos que a imagem também é construída a partir de outros componentes avaliativos. Assim, além de considerar a percepção dos turistas sobre o evento e a sua marca, é fundamental que os gestores compreendam como os residentes se apropriam do evento, para que possam fortalecer o pertencimento da comunidade local. No turismo, é razoável prever que o apego dos residentes a um evento, um importante fator de atitude, afetará positivamente seu comportamento perante o evento. Uma identidade de marca ajustada à 
identidade do consumidor prediz um relacionamento a longo prazo dos indivíduos com a marca (Berrozpe, Campo \& Yagüe, 2019). Porém, cabe verificar se os efeitos positivos que podem influenciar o senso de pertencimento dos residentes variariam de acordo com os diferentes níveis de autoeficácia do que é entregue aos residentes.

Ainda, uma marca, segundo Keller e Machado (2006) reúne dimensões racionais e tangíveis, e dimensões simbólicas, emocionais e intangíveis. Os elementos simbólicos são fruto dos estímulos que criam associações na mente do consumidor, e podem ser o nome, slogan, logotipo, letras, mascotes ou personagens, entre outros elementos (Kladou, Kavaratzis, Rigopoulou \& Salonika, 2017). Os elementos de marca, como as mascotes, fazem parte das estratégias da comunicação do marketing que, dentre outras ações, buscam meios de promover suas marcas construindo a ligação com seus consumidores (Caufield, 2012; Weszka, 2011).

A Feira Nacional do Doce - FENADOCE - é realizada no município de Pelotas (RS), Brasil, desde 1986, com o objetivo de divulgar a cultura doceira e desenvolver o turismo no município, o qual possui reconhecida tradição doceira (De Figueiredo \& Cavedon, 2012; Magalhães, 1993; Ferreira, Cerqueira \& Rieth, 2008). Tradição advinda do período áureo dos charqueadores portugueses que tinham o hábito de servirem doces finos nos encontros da alta sociedade pelotense e, também, dos doces coloniais de frutas dos imigrantes do período do declínio das charqueadas (Berselli, Tricárico \& Rossini, 2019). O festival ocorre anualmente durante 19 dias, e recebe aproximadamente 300 mil pessoas em cada edição. Em 2007 os gestores do evento criaram uma mascote inspirada nos projetos da Cow Parade (Nova Iorque) e nos Leões de Munique (Alemanha), para representar a cultura doceira do município (Berselli et al., 2019). Formigas estilizadas de 1,90m de altura, como aquelas retratadas na Figura 1, foram espalhadas pelos principais prédios históricos e pontos turísticos de Pelotas para fortalecer os laços da comunidade com o evento, e a partir dali a formiga passou a ser apresentada como mascote oficial do evento.

Figura 1. Figura digital (a) e estátua da mascote do evento (b)

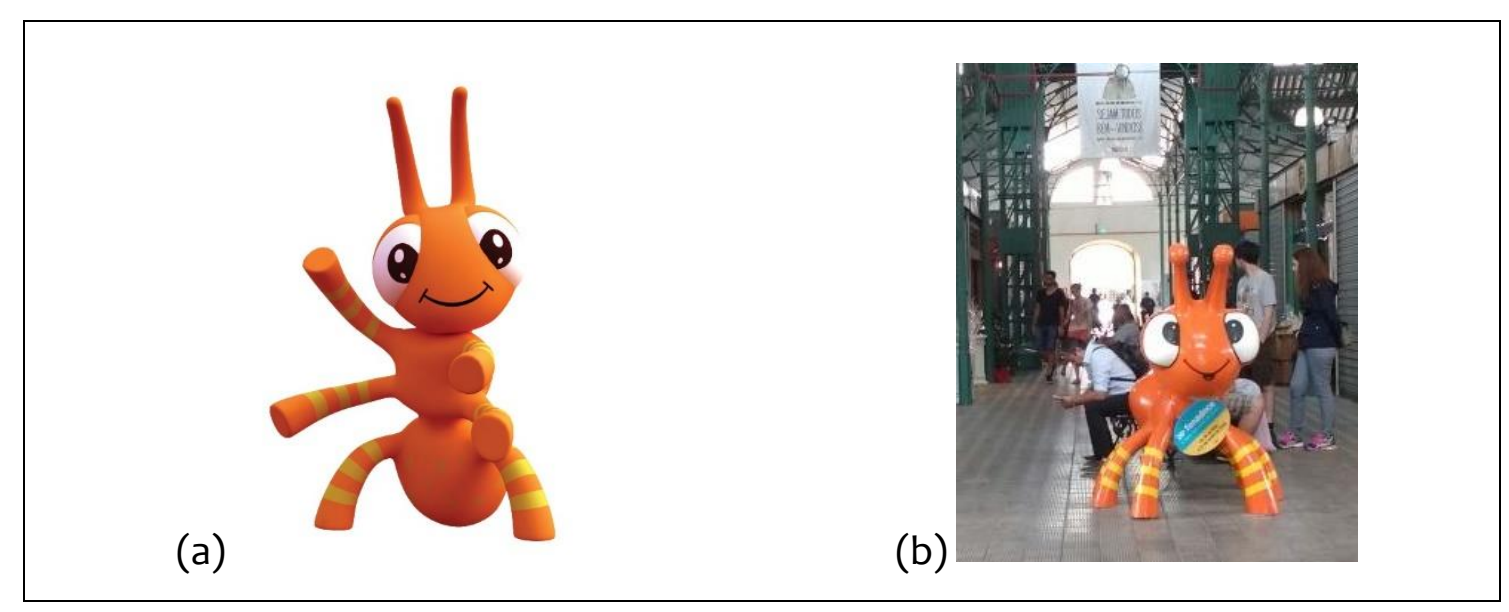

Fontes: (a) Agência Incomum; (b) Acervo próprio (2018). 
A pesquisa se justifica pela importância que têm os festivais para a promoção da cultura e preservação do patrimônio cultural (Dos Santos, Zucco \& Kraus, 2015), assim como pela relevância que tem o papel dos residentes na sustentabilidade dos destinos (López, Virto, Manzano \& Miranda, 2018; Nunkoo \& Gursoy, 2012; Zhang \& Xu, 2019). Tendo em vista que modelos de estudos mais abrangentes e integrativos ainda são escassos (Zhang \& Xu, 2019), o presente trabalho analisa a FENADOCE para entender como os residentes avaliam a qualidade da organização do evento, os benefícios e os inconvenientes associados ao festival, como eles se apropriam do festival, e que relação estabelecem com a formiga, enquanto mascote-símbolo.

$\mathrm{Na}$ literatura revisada não foi encontrada qualquer referência a estudo de imagem e marca de eventos envolvendo a relação organização - impactos (positivo e negativos) percebidos - apropriação do evento - apropriação da marca/mascote, especialmente no contexto brasileiro. O presente estudo busca responder: (1) se/como a qualidade da organização do evento contribui para ampliar/minorar os impactos positivos e negativos percebidos pelos residentes; (2) se/como a qualidade da organização do evento contribui para aumentar/diminuir o sentido de pertencimento o evento pelos moradores, e (3) se/como os impactos positivos e negativos percebidos associados ao evento contribuem para a apropriação do evento e da mascote por parte dos residentes. Trata-se de um estudo descritivo de natureza quantitativa, baseado em dados primários levantados diretamente no local do evento, que busca entender o processo de criação de valor para os residentes, a partir da organização do evento e das externalidades criadas por ela.

\section{Revisão da literatura}

\subsection{A relação entre imagem de eventos e festivais e impactos percebidos}

Eventos e festivais têm se tornado cada vez mais importantes no setor do turismo (Getz et al., 2010). A literatura identifica benefícios econômicos, sociais e culturais na realização de eventos especiais e festivais (Akhoondnejad, 2016; Gursoy et al., 2004). Porém se faz necessário um aprofundamento da compreensão sobre a imagem dos eventos e festivais (Lai, 2018; Deng, Li \& Shen, 2013) nas pesquisas. A imagem de um evento é resultado da avaliação de diversos componentes que influenciam o comportamento (Deng et al., 2013).

A literatura de branding explica que a imagem de um produto/serviço é formada mentalmente pelo consumidor a partir do que ele ganha com sua aquisição (Magalhães, 2020). Ou seja, a imagem é formada a partir da qualidade da sua performance. $O$ conceito de imagem seria o resultado do capital da imagem e, para a sua construção positiva, a identidade de marca deve considerar uma proposta de valor que produza benefícios funcionais, emocionais e de autoexpressão (Magalhães, 2020; Aaker, 2001). Além disso, o conceito de marketing experiencial diz que as decisões de compra são relacionadas à promessa de uma experiência (Healey, 2009).

No turismo, Rola et al. (2018) destacaram que as marcas de destino têm uma dimensão representacional e uma dimensão funcional. Neste sentido, a marca seria o resultado de atributos de autoexpressão do indivíduo e dos utilitários do destino (Rola et al., 2018). Lai (2018) afirmou que a imagem de evento deve ter uma estrutura multidimensional semelhante 
à da imagem de destino, portanto, supõe-se que eventos e festivais tenham presentes os mesmos atributos. Ainda, no contexto dos residentes, a elaboração de uma imagem condizente com sua identidade é imprescindível. Assim, elabora-se uma imagem coerente e uma marca forte.

Para Deng et al. (2013), a imagem de um evento é mensurada por aspectos ligados aos benefícios, instalações, serviço, tema e conteúdo do evento, permitindo-se avaliar as impressões cognitivas e afetivas da comunidade. Para Lai (2018), a imagem de evento é resultado de influência de fatores internos e externos, dentre eles, a identificação com o evento, ajuste de marca e familiaridade com slogans, tipos de turistas e do destino, idade e gênero, objetivo da viagem, entre outros. Para Chiang, Xu, Kim, Tang e Manthiou (2017), os festivais são atividades únicas, que fomentam a identidade e revitalizam atrações estagnadas. Para além disso, são capazes de conservar e celebrar a cultura, fomentar as redes sociais e acelerar as economias. Prayag, Hosany, Nunkoo e Alders (2013) identificaram que os impactos econômicos e socioculturais percebidos positivos e negativos influenciaram na atitude geral dos residentes. Rola et al. (2018) afirmaram que o impacto turístico nos destinos está associado à sua imagem. Assim, é possível compreender que os impactos percebidos pelos residentes no que condiz a ganhos e custos sentidos durante a experiência com o evento influencia diretamente na imagem formada na mente deste consumidor.

Entre os principais benefícios proporcionados aos residentes encontram-se a melhora da qualidade de vida, a promoção do entretenimento e dos relacionamentos pessoais, as oportunidades de emprego e o melhoramento da infraestrutura, que revitalizam tradições e aumentam o apego ao lugar (Grappi \& Montanari, 2011; Gursoy et al., 2004). Adicionalmente, em muitas ocasiões o apego ao local pode ser mais importante para a escolha de um evento, do que o evento em si (Queiroz, Carvalho, Macedo \& Gosling, 2019). Ademais, Gu e Ryan (2008) propõem que a atitude dos residentes não seja explicada apenas em termos de custos e benefícios percebidos, mas em termos de sistemas de valores pessoais da identidade, o que traria uma análise perceptual mais completa.

Isto posto, é possível assumir que a qualidade da organização do evento embasa o processo de criação de valor (benefícios funcionais, emocionais e de autoexpressão) para turistas e residentes, e por isso é um elemento-chave para o sucesso ou fracasso do evento. Portanto, conforme a revisão de literatura apresentada, espera-se que:

H1: Quanto maior for a qualidade percebida na organização do evento, maiores serão os benefícios socioculturais associados a ele.

H2: Quanto maior for a qualidade percebida na organização do evento, mais forte será o sentimento de pertença dos moradores da localidade.

H3: Quanto maior for a qualidade percebida na organização do evento, maiores serão os benefícios socioeconômicos associados a ele.

H4: Quanto maior for a qualidade percebida na organização do evento, maior será a imagem positiva da mascote.

H5: Quanto maior for a qualidade percebida na organização do evento, menores serão os efeitos negativos percebidos pelos residentes. 


\subsection{O senso de pertencimento pela identidade social}

Para Wang e Chen, (2015) e Gu e Ryan (2008) a atitude dos residentes em relação ao turismo não é explicada apenas em termos de custos e benefícios percebidos, mas em termos de sistemas de valores pessoais com o destino. Alguns estudos têm buscado entender através da identidade social, aspectos sociopsicológicos dos residentes na atitude geral do desenvolvimento do turismo (Palmer, Koenig-Lewis \& Jones, 2013). A Teoria da Identidade Social (TIS) de Tajfel (1978) é compreendida como o autoconceito do indivíduo resultante da associação em grupos sociais, do valor e do significado emocional resultantes dessa interação. Ou seja, a identidade se constrói pela referência aos outros, expressando as similaridades de alguns grupos (Haobin Ye, Qiu Zhang, Huawen Shen \& Goh, 2014; Palmer et al., 2013).

Como resultado, residentes que se identificam e se sentem pertencentes a um grupo de valores compartilhado tendem a ter orgulho, senso de pertencimento, apego/lealdade, maior comprometimento, imagem positiva e autoestima (adaptado de Berrozpe et al., 2019; Palmer et al., 2013; Wang, Zhou, Lee \& King, 2014). Além disso, segundo Berrozpea et al. (2019) e Jackson (2002) esta dimensão é a única capaz de mensurar uma percepção no qual os sujeitos estão cientes de fazerem parte de um grupo existente e que se sentem parte deste grupo. Mais que isso, Berrozpea et al. (2019) afirmam que apenas a TIS tem a capacidade de capturar e revelar a unidade psicológica entre identidade da marca e identidade do consumidor. A TIS consegue mensurar o relacionamento a longo prazo com os consumidores e causa consequências positivas como: satisfação, confiança, comprometimento, tendência a compra, intenção de compra, compromisso com a marca, boca-a-boca positiva, resistência a informações negativas e avaliação positiva do consumidor (Berrozpea et al., 2019).

No turismo, o estudo de Choo, Park e Petrick (2011) revelou que quanto mais os moradores se identificaram positivamente com sua marca do destino, maior é a propensão à sua participação no turismo e atividades de lazer, participar de atividades para satisfação dos visitantes, e suas intenções boca-a-boca positivas. Small (2007) identificou fatores ligados aos aspectos positivos como o senso de identidade e união da comunidade relacionado à demonstração de singularidade que o festival proporciona, oportunidades de entretenimento, socialização, crescimento e desenvolvimento da comunidade. São atributos que permitem aos membros da comunidade sentirem senso de identidade e conectividade, que poderiam ocasionar segundo o autor sentimentos de união e de posse por promover sentimento de orgulho nos moradores, portanto, caberia mensurar se estes benefícios socioculturais contribuem num maior senso se pertencimento por auto identificação. Ainda, para Palmer et al. (2013) as atitudes positivas dos moradores em relação ao turismo estão relacionadas ao sentido de pertencimento e ao envolvimento com as atividades de turismo; e o contrário pode acontecer na falta de identificação com o destino, que poderá criar obstáculos à atividade. Portanto, espera-se:

H6: Os benefícios socioculturais atribuídos ao festival favorecem o senso de pertencimento dos residentes pelo festival. 
Alguns estudos relacionaram a TIS com os estudos sobre marca (Bagozzi \& Dholakia, 2006; Tuškej, Golob \& Podnar, 2013), uma vez que clientes são atraídos por produtos e serviços ligados à sua identidade social. A TIS define o nível de identificação da marca do consumidor com o grau em que a marca expressa e melhora a identidade dos consumidores (Kim, Jun, Walker \& Dran, 2015). Nesta perspectiva, a identificação com a marca está intrinsicamente ligada a identidade do consumidor (Berrozpe et al., 2019). No âmbito do consumo, a TIS pode explicar como um produto ou serviço é avaliado ou escolhido de acordo com o mérito ou a virtude que nele é vista (Chiang et al., 2017). Constataram que a identificação dos visitantes de um festival é influenciada pelos valores funcionais (qualidade, personalização, performance do suporte de serviços) e valores experienciais (sentimentos e emoções, entretenimento, consumo emocional).

Embora os festivais resgatem tradições, identidades e culturas, estudos na área de turismo relacionados a residentes tem encontrado que geralmente há uma maior percepção dos beneficios econômicos em relação aos demais (Uysal, Sirgy, Woo \& Kim, 2016). Principalmente porque os beneficios económicos podem demonstrar serem um benefício de maior prazo do que a experiência ocorrida no momento do evento. Portanto, a identidade social influencia a maneira como os eventos e festivais serão avaliados, positivamente ou não. Pela literatura revisada, se os benefícios econômicos são a percepção mais relevante perante os residentes inferimos que ele favorece a um maior senso se pertencimento ao festival. Portanto espera-se que:

H7: Os benefícios socioeconômicos atribuídos ao festival favorecem o senso de pertencimento dos residentes pelo festival.

\subsection{A marca e as mascotes}

A marca é uma entidade representativa, e como tal deve comunicar claramente a personalidade, o caráter e a natureza daquilo que representa (Weszka, 2011). Os elementos de marca são definidos como dispositivos comerciais que servem para identificar e diferenciar a marca (Keller, Apéria, \& Georgson, 2008; Weszka, 2011), como por exemplo, os nomes, logos e símbolos, personagens, slogans e jingles. As mascotes ajudam as marcas a ganhar vida com qualidades humanas, sejam elas emoções, pensamentos e personalidade (Hoolwerff, 2014; Weszka, 2011). Os mascotes ou personagens são um recurso para personificar uma marca, transmitindo uma imagem mais agradável e elevar o nível da marca para não ser apenas um logotipo (Caufield, 2012). A criação de personagens favorece a consolidação da marca (Garretson \& Niedrich, 2004), na medida que possibilita a criação de associações favoráveis ao evento (Weszka, 2011). A criação de uma mascote para atuar como "embaixadora" ajuda a marca a ganhar vida e facilita a sua memorização pelo público (Hoolwerff, 2014).

Além disso, partindo-se da premissa que as representações sociais reúnem um conjunto de símbolos relacionados às crenças, imagens, memórias coletivas, práticas etc., que fazem parte do senso de lugar (de Rosa, Bocci \& Dryjanska, 2019), entende-se o poder simbólico de representações sociais como a identidade, a imagem e demais elementos de marca e sua 
importância para o branding do festival. Poucas são as pesquisas sobre mascotes no turismo. Um desses exemplos é a pesquisa de Weszka (2011) realizou um estudo sobre como os elementos de marca foram trabalhados pelo mega-evento FIFA Copa do Mundo com os destinos anfitriões dos eventos dos anos 2002 na Coréia/Japão, 2006 na Alemanha e 2008 na África do Sul. $\mathrm{Na}$ análise dos três mascotes, somente o leopardo Zakumi, representou intimamente a identidade, representou uma inspiração local por meio de um conceito bem elaborado (Weszka, 2011).

As associações de marcas possuem três categorias - atributos, benefícios e atitudes (Keller, 1998; Qu, Kim \& Im, 2011). Os atributos são o que a marca é e o que oferece, ou seja, o que esta envolvido com sua compra; os benefícios são os valores anexos funcionais, simbólicos e experiências, ou seja, o que mais a marca oferece como "plus" e, por fim, as atitudes são a resposta do consumidor à marca, suas avaliações que resultam em escolhê-la (Qu et al., 2011).

O envolvimento com o festival, enquanto produto turístico, desperta nos moradores locais um sentimento de pertencimento recíproco, como se fossem um pouco proprietários do evento ao mesmo tempo. A mascote da Fenadoce foi concebida para personificar e representar a marca do evento e estabelecer laços afetivos com seus visitantes. Se estimamos que a mascote representa a Fenadoce, ela deve representar os seus atributos e benefícios perante a percepção dos residentes. Assim como espera-se uma transferência do senso se pertencimento do evento à sua personificação de marca. Logo, inferimos que:

H8: Os benefícios socioculturais do festival favorecem a avaliação da imagem da mascote.

H9: O senso de pertencimento ao evento favorece a avaliação da imagem da mascote.

Portanto, os fatores diferenciais de uma marca podem ser tangíveis ou racionais, relacionados ao produto em si e seu desempenho, e fatores intangíveis ou simbólicos/emocionais, relacionados ao que a marca representa, sua reputação (Keller \& Machado, 2006). Elementos de marca podem assumir um papel principal na criação de consciência do produto ou destino turístico (Hem \& Iversen, 2004). Por isso, é possível que tanto a marca quanto a mascote do evento sejam avaliadas através de componentes racionais (tangíveis) e componentes simbólicos (emocionais e intangíveis) (Keller et al., 2008). Os benefícios socioeconômicos podem ser um fator importante para os moradores, se há uma percepção sobre este benefício proporcionado pelo evento, logo espera-se que a sua personificação também englobe tal valor funcional/tangível. Por outro lado, qualquer impacto negativo que venha a ser percebido, compreendido como uma falha de desempenho da marca, pode influenciar negativamente à imagem da mascote. Por isso espera-se que:

H10 - Os benefícios socioeconômicos do festival favorecem a avaliação da imagem da mascote. 
H11 - Os impactos negativos influenciam negativamente a avaliação da imagem da mascote.

Figura 2. Síntese do modelo teórico

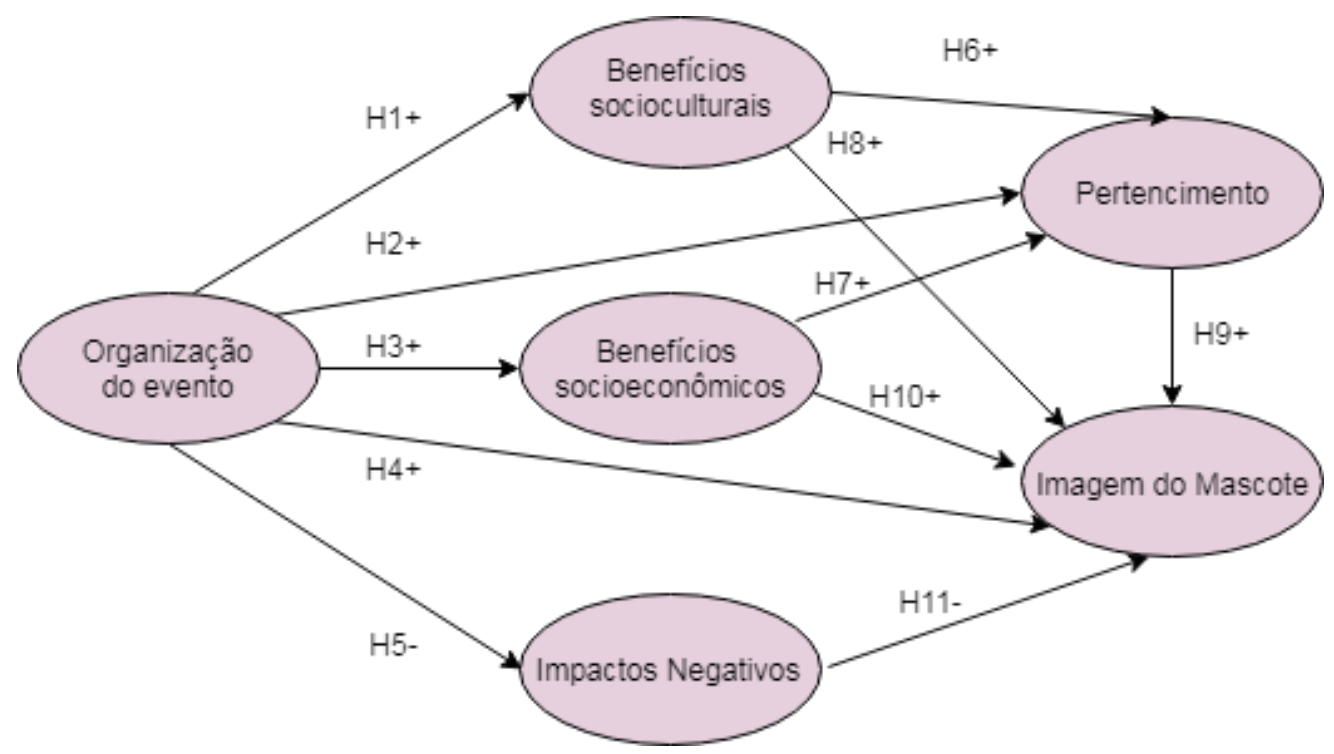

\section{Metodologia da pesquisa}

A presente pesquisa é de natureza aplicada, com objetivo exploratório-descritivo, e utiliza uma abordagem predominantemente quantitativa, precedida de uma etapa exploratória para delimitação do elenco de variáveis de interesse. O caráter descritivo da pesquisa se justifica por envolver a descrição das características da população, do fenômeno e das relações entre ambos, utilizando técnicas padronizadas de coleta de dados, com menor interferência do pesquisador.

O instrumento utilizado no levantamento dos dados foi inspirado em estudos anteriores a respeito do tema (Chiang et al., 2017; Delamere, 2001; Deng et al., 2015; Gursoy et al., 2004; Hem \& Iversen, 2004; Prayag et al., 2013; Small, 2007), realizados em situações correlatas. O questionário foi elaborado com base na revisão teórica, a qual permitiu antever a existência de ao menos três dimensões de análise: a organização do evento enquanto produto turístico e a percepção dos impactos nos residentes; a apropriação do evento ao ideário afetivo, como causa de orgulho ou de pertencimento da população; e a imagem da mascote, como representação da marca e sua congruência com a identidade coletiva. Tais elementos foram representados por 35 questões fechadas organizadas por meio de escala tipo likert de sete pontos de concordância, variando de um para "discordo totalmente" a sete para "concordo totalmente"; e a imagem da mascote foi representada por 10 questões de diferencial semântico.

O modelo de estudo foi desenhado a partir das hipóteses construídas, considerando os insights levantados na fase exploratória. Os dados foram colhidos diretamente no local do evento, entre os dias 30/Mai/2018 e 15/Jun/2018, através de formulários impressos, e 
avaliados em conjunto por todos os autores. Dos 482 questionários aplicados, 424 foram considerados válidos para fim de análise. Os respondentes foram escolhidos por conveniência, entre residentes da cidade de Pelotas que visitaram a FENADOCE no ano de 2018. A Análise Fatorial Exploratória - AFE foi realizada com o pacote IBM-PASW Statistics 18, e os modelos confirmatórios (estrutural e de mensuração) foram analisados com o software AMOS 21.0.

\section{Apresentação e análise dos resultados}

\subsection{Perfil da amostra}

Os resultados apontam que a maioria da amostra levantada possui ensino superior e corresponde à faixa de idade entre 18 e 30 anos (47,9\%), e a menor participação corresponde aos maiores de 60 anos ( $8,7 \%)$. Sobre o nível de escolaridade, a maior parte dos respondentes possuem grau de Pós-graduação (25,2\%) e Superior Incompleto (24,8\%). Quanto ao estado civil dos respondentes, a maior amplitude de respondentes se declarou solteiros $(52,6 \%)$ seguidos de respondentes declarados casados (37,7\%). Por se tratar de uma amostra colhida por conveniência, é possível que as proporções não sejam as mesmas da população local.

\subsection{Análise fatorial exploratória}

Como não se conhecia previamente a composição de cada fator, os dados foram examinados preliminarmente através de Análise Fatorial Exploratória (AFE), cujo resultado sugeriu a existência de oito fatores. A AFE foi realizada como procedimento preparatório para a Análise Fatorial Confirmatória (AFC), e por essa razão a confiabilidade e a validade dos fatores identificados será discutida apenas na etapa seguinte. Os fatores e as variáveis correspondentes encontram-se na Tabela 1.

Tabela 1. Composição preliminar dos fatores (identificada por AFE)

\begin{tabular}{l|l}
\hline \multicolumn{1}{c|}{ Fatores/Variáveis } & Cargas Fatoriais \\
\hline \multicolumn{1}{c}{ Benefícios Socioculturais } & \\
\hline V2 - Impacto cultural positivo & 0,754 \\
\hline V4 - Motivo de orgulho & 0,693 \\
\hline V1 - Fortalece a identidade da minha comunidade & 0,668 \\
\hline V3 - Aproxima as pessoas que moram no destino & 0,568 \\
\hline V8 - Pelotas promovida como destino turístico com a ajuda da Fenadoce & 0,537 \\
\hline V9 - Ajuda a preservar a cultura local & 0,526 \\
\hline V6 - Mostra a comunidade como única * & 0,336 \\
\hline \multicolumn{1}{c}{ Benefícios Socioeconômicos } & 0,795 \\
\hline V18 - Promove oportunidade de entretenimento & 0,788 \\
\hline V14 - Oferece atividades de recreação familiares & 0,726 \\
\hline V19 - Permite boas experiências culturais & 0,482 \\
\hline V16 - Proporciona oportunidades de empregos locais & \\
\hline
\end{tabular}




\begin{tabular}{|c|c|}
\hline Fatores/Variáveis & Cargas Fatoriais \\
\hline V15 - A prestação de serviços públicos e de infraestrutura melhoram** & 0,456 \\
\hline \multicolumn{2}{|l|}{ Impactos Negativos } \\
\hline V22 - Qualidade de vida prejudicada (inv) & 0,817 \\
\hline V23 - Excesso de pessoas traz problemas para a cidade (inv) & 0,779 \\
\hline V21 - Rotina alterada de forma negativa (inv) & 0,758 \\
\hline V26 - Aumenta impostos pagos pela população da cidade (inv) & 0,634 \\
\hline \multicolumn{2}{|l|}{ Qualidade percebida da Organização do Evento } \\
\hline V30 - Possui banheiros convenientes e limpos & 0,769 \\
\hline $\begin{array}{l}\text { V29 - Os locais para alimentação (comidas e bebidas) são suficientes e } \\
\text { convenientes }\end{array}$ & 0,747 \\
\hline V31 - A equipe do evento oferece serviços de alta qualidade & 0,738 \\
\hline V33 - Os funcionários do evento são profissionais e bem preparados ** & 0,738 \\
\hline V32 - Possui boa segurança & 0,703 \\
\hline V28 - As áreas de descanso são suficientes e convenientes & 0,568 \\
\hline \multicolumn{2}{|l|}{ Senso de Pertencimento } \\
\hline V10 - Quando alguém critica a Fenadoce, parece um insulto pessoal & $-0,867$ \\
\hline V12 - Para mim, quando alguém elogia a Fenadoce, parece elogio pessoal & $-0,847$ \\
\hline V13 - Quando a mídia critica a Fenadoce, eu me sinto envergonhado & $-0,830$ \\
\hline V11 - Quando falo sobre a Fenadoce, costumo dizer nós ao invés de eles & $-0,758$ \\
\hline V7 - Eu me interesso pelo que os outros pensam sobre a Fenadoce & $-0,579$ \\
\hline V5 - O sucesso da Fenadoce é o meu sucesso & $-0,564$ \\
\hline \multicolumn{2}{|l|}{ Benefício/Custo** } \\
\hline V34 - O estacionamento disponível aos visitantes é apropriado ** & 0,765 \\
\hline V35 - O preço do ingresso é apropriado ** & 0,632 \\
\hline \multicolumn{2}{|l|}{ Aspectos Objetivo-Funcionais da Mascote } \\
\hline V36 - Como símbolo: negativo-positivo & 0,655 \\
\hline V40 - Similaridade (produto turístico): não representativo-muito representativo & 0,577 \\
\hline V38 - Como símbolo: nada familiar-muito familiar & 0,507 \\
\hline $\begin{array}{l}\text { V17 - A reputação nacional de Pelotas por meio da exposição da mídia melhora } \\
\text { com a Fenadoce ** }\end{array}$ & 0,404 \\
\hline \multicolumn{2}{|l|}{ Aspectos Afetivos da Mascote } \\
\hline V43 - Design: desequilibrado-equilibrado & 0,877 \\
\hline V42 - Design: desatualizado-atualizado & 0,864 \\
\hline V44 - Design: ineficaz-eficaz & 0,848 \\
\hline V45 - Design: falso-autêntico & 0,847 \\
\hline V41 - Design: confuso-simples & 0,650 \\
\hline V37 - Como símbolo: pouco atraente-atraente * & 0,580 \\
\hline V39 - Como símbolo: não reconhecível-facilmente reconhecível * & 0,522 \\
\hline
\end{tabular}

Fonte: Dados da pesquisa. Observações: * Variáveis realocadas, no modelo confirmatório. ** Variáveis excluídas do modelo confirmatório. 


\subsection{Análise fatorial confirmatória e modelagem de equações estruturais}

O modelo de mensuração foi construído a partir dos fatores identificados na AFE (Tabela 2), os quais foram reespecificados respeitando-se o princípio do alinhamento teórico (Brei \& Liberali Neto, 2006). Seis, dos sete fatores indicados pela AFE, foram mantidos com nomenclatura adaptada ao contexto do festival e um - a relação benefício/custo - precisou ser excluído do modelo por baixa confiabilidade. A imagem da mascote manteve a característica dual, com uma faceta objetiva (valor econômico) e outra subjetiva (valor simbólico), para melhor representar a percepção dos residentes acerca daquele símbolo. A validade discriminante entre as duas facetas será discutida adiante.

O modelo reespecificado apresentou RMSEA inferior ao máximo esperado de 0,06 $\mathrm{Hu}$ \& Bentler, 1999), e os índices de IFI, TLI e CFI apresentaram valores próximos a 0,95, o que segundo Brown (2006: 87) indicam "razoável grau de ajustamento". Todas as cargas fatoriais superaram o dobro do erro padrão correspondente, o que atesta a validade convergente do modelo (Anderson \& Gerbing, 1988). Por economia de espaço, o modelo de mensuração será retratado em conjunto com o modelo estrutural, no próximo item.

A validade discriminante, que verifica o quanto cada fator da escala é suficientemente diferente dos demais, foi avaliada inicialmente pelo método de Fornell e Larcker (1981). Conforme se vê na Tabela 2, com exceção do par formado pelos fatores 6 e 7, todos os demais atenderam ao padrão recomendado por F\&L (raiz quadrada da AVE maior do que as correlações entre o próprio fator e os demais).

Tabela 2. Confiabilidade composta, variância média extraída e validade discriminante

\begin{tabular}{lccccccc}
\hline \multicolumn{1}{c}{ Fatores } & $(1)$ & $(2)$ & (3) & (4) & (5) & (6) & (7) \\
\hline Organização (1) & $\mathbf{0 , 7 6 1}$ & & & & & & \\
Benefícios Socioculturais (2) & 0,470 & $\mathbf{0 , 7 5 4}$ & & & & & \\
Benefícios Socioeconômicos (3) & 0,607 & 0,741 & $\mathbf{0 , 7 2 1}$ & & & & \\
Pertencimento (4) & 0,494 & 0,635 & 0,536 & $\mathbf{0 , 7 2 1}$ & & & \\
Impactos Negativos (5) & 0,187 & 0,088 & 0,113 & 0,092 & $\mathbf{0 , 8 1 6}$ & & \\
Mascote - Valor Econômico (6) & 0,268 & 0,161 & 0,218 & 0,151 & 0,163 & $\mathbf{0 , 7 2 1}$ & \\
Mascote - Valor Simbólico (7) & 0,285 & 0,169 & 0,194 & 0,142 & 0,210 & 0,799 & $\mathbf{0 , 5 9 1}$ \\
\hline Confiabilidade Composta (CR) & 0,831 & 0,855 & 0,798 & 0,874 & 0,856 & 0,831 & 0,832 \\
Variância Média Extraída (AVE) & 0,580 & 0,569 & 0,520 & 0,520 & 0,667 & 0,519 & 0,349 \\
\hline
\end{tabular}

Como a correlação entre os fatores (6) e (7) superou ligeiramente as AVEs de um e de outro, a similaridade entre eles foi avaliada também pelo critério de Bagozzi, Yi e Phillips (1991). Por este critério, reconhece-se a discriminação entre dois fatores, se houver degradação significativa do modelo após fixar-se a covariância entre eles em um (1). Conforme visto na Tabela 3 , o modelo restringido apresentou ajustamento significativamente menor (diferença de $\chi^{2}=67,2$; diferença de $G L=1$; Sig. = 0,000) que 0 modelo estimado livremente, confirmando que os fatores não são perfeitamente correlacionados, ou seja, que há validade discriminante também entre estes. 


\subsection{Modelagem de equações estruturais}

As relações entre os construtos foram estimadas através de um Modelo de Equações Estruturais, a partir do Modelo de Mensuração, para avaliar as diversas hipóteses de pesquisa. Para facilitar a leitura, os impactos negativos do evento foram revertidos e introduzidos no modelo pelo valor inverso (INV), tornando assim positivas as suas relações com os outros fatores. O modelo completo apresentou índices aceitáveis de ajustamento, conforme ilustrado na primeira linha da Tabela 3.

Tabela 3. Índices de ajustamento geral do modelo

\begin{tabular}{|c|c|c|c|c|c|c|c|c|}
\hline Modelos & CMIN & DF & $\mathrm{P}$ & CMIN/DF & IFI & TLI & CFI & RMSEA \\
\hline Estimado livremente & 1184,3 & 638 & 0,000 & 1,856 & 0,923 & 0,915 & 0,923 & 0,045 \\
\hline Modelo restringido ${ }^{1}$ & 1251,6 & 639 & 0,000 & 1,959 & 0,914 & 0,905 & 0,913 & 0,048 \\
\hline Diferenças de $\chi^{2}$ & 67,2 & 1 & 0,000 & - & - & - & - & - \\
\hline
\end{tabular}

A Figura 3 apresenta as relações testadas e seus valores padronizados. Para facilitar o entendimento da figura, as relações sem significância estatística $(P<0,05)$ foram substituídas por setas pontilhadas. $O$ resultado confirmou que a organização do evento é a principal propulsora de valor para os residentes, tendo em vista a sua capacidade de influenciar significativamente todos os demais fatores. Conforme se vê na figura e na Tabela 4, a [boa] organização do evento favorece positiva e significativamente os benefícios socioculturais percebidos $\left(\mathrm{H}_{1}\right)$, o sentido de pertencimento $\left(\mathrm{H}_{2}\right)$, os benefícios socioeconômicos $\left(\mathrm{H}_{3}\right)$, as duas facetas da imagem da mascote $\left(\mathrm{H}_{4}\right)$ e a reversão de impactos negativos ( $\left.\mathrm{H}_{5}\right)$, confirmando assim as cinco primeiras hipóteses de pesquisa.

\footnotetext{
${ }^{1}$ Modelo restringido, com covariância entre o "valor econômico" e o "valor simbólico" da mascote forçada a 1 (um), para avaliação da validade discriminante (procedimento de Bagozzi \& Phillips, 1982; Bagozzi, Yi, \& Phillips, 1991)
} 
Figura 3. Modelo final

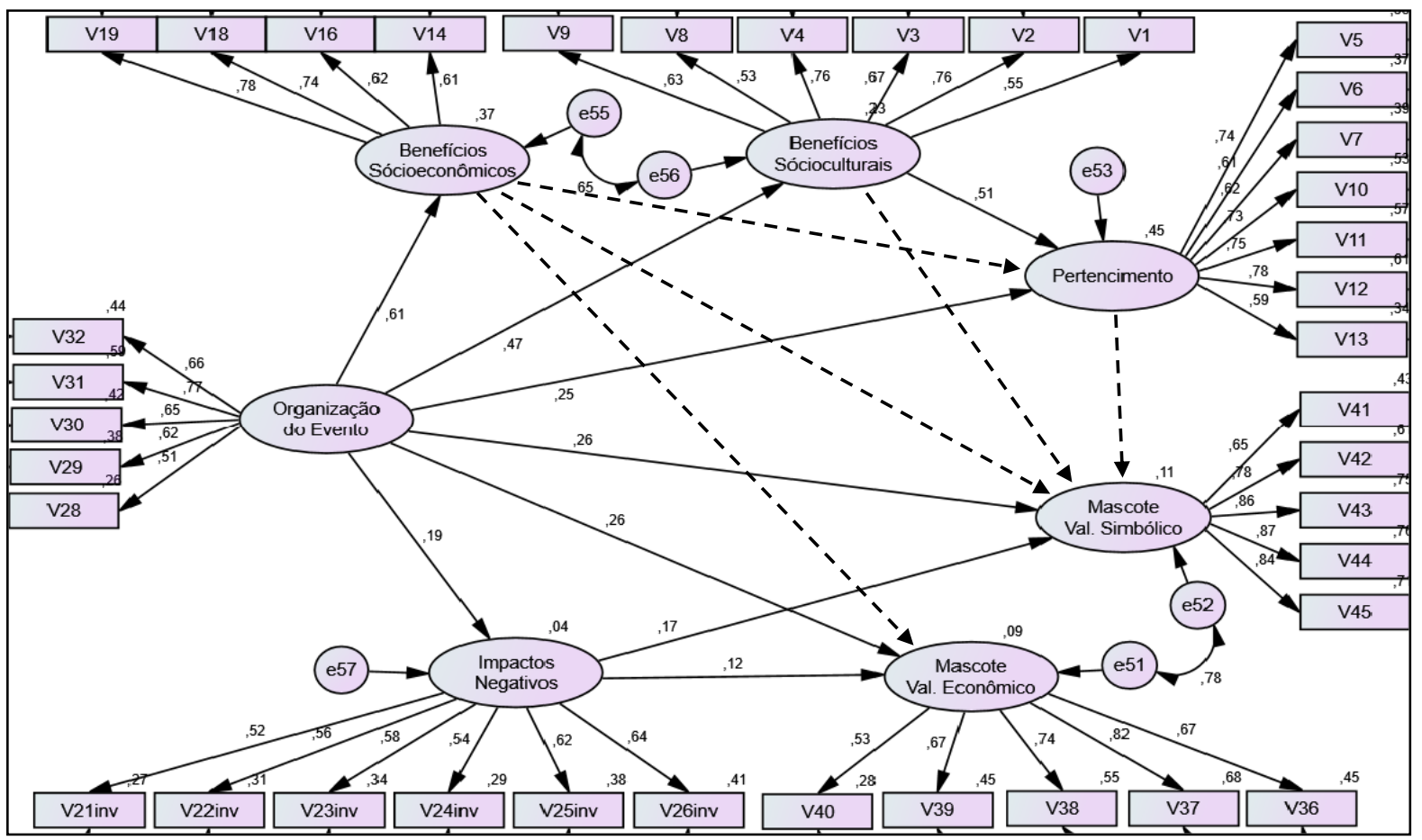

O resultado também confirma a existência de influência dos benefícios socioculturais na construção do pertencimento (H6), mas não confirma a esperada influência dos benefícios socioeconômicos naquele construto $(\mathrm{H} 7)$. Como esperado, confirma-se a influência dos impactos negativos nas duas facetas da imagem da mascote (H11), mas não se confirmam a influência dos benefícios socioeconômicos (H10), nem dos benefícios socioculturais (H8), nem do pertencimento ( $\mathrm{H} 9)$ no valor simbólico da mascote. Em resumo, confirmam-se as hipóteses $\mathrm{H}_{1}, \mathrm{H}_{2}, \mathrm{H}_{3}, \mathrm{H} 4, \mathrm{H} 5, \mathrm{H} 6$ e H11, e não se confirmam as hipóteses H7, H8, H9 e H10.

Tabela 4. Relações estruturais confirmadas

\begin{tabular}{|c|c|c|c|c|c|c|c|}
\hline \multicolumn{3}{|c|}{ Relações Estruturais } & \multirow{2}{*}{$\begin{array}{c}\begin{array}{c}\text { Cargas } \\
\text { Originais }\end{array} \\
0,372\end{array}$} & \multirow{2}{*}{$\frac{\text { S.E. }}{0,063}$} & \multirow{2}{*}{$\frac{P}{0,000}$} & \multirow{2}{*}{$\begin{array}{c}\begin{array}{c}\text { Cargas } \\
\text { Padroniz. }\end{array} \\
0,48\end{array}$} & \multirow{2}{*}{$\begin{array}{c}\mathrm{H} \\
\mathrm{H} 1\end{array}$} \\
\hline Organização & $\rightarrow$ & Benefic. Socioculturais & & & & & \\
\hline Organização & $\rightarrow$ & Pertencimento & 0,357 & 0,090 & 0,000 & 0,25 & $\mathrm{H}_{2}$ \\
\hline Organização & $\rightarrow$ & Benefic. Socioeconôm. & 0,630 & 0,087 & 0,000 & 0,61 & $\mathrm{H}_{3}$ \\
\hline Organização & $\rightarrow$ & Mascote Val. Simbólico & 0,203 & 0,050 & 0,000 & 0,26 & $\mathrm{H} 4 \mathrm{a}$ \\
\hline Organização & $\rightarrow$ & Mascote Val. Econômico & 0,157 & 0,041 & 0,000 & 0,26 & $\mathrm{H} 4 \mathrm{~b}$ \\
\hline Organização & $\rightarrow$ & Impactos Negativos & 0,214 & 0,078 & 0,006 & 0,19 & $\mathrm{H}_{5}$ \\
\hline Benefíc. Socioculturais & $\rightarrow$ & Pertencimento & 0,928 & 0,131 & 0,000 & 0,51 & $\mathrm{H} 6$ \\
\hline Impactos Negativos & $\rightarrow$ & Mascote Val. Simbólico & 0,118 & 0,042 & 0,005 & 0,17 & H11a \\
\hline Impactos Negativos & $\rightarrow$ & Mascote Val. Econ. & 0,065 & 0,034 & 0,056 & 0,12 & $\mathrm{H} 11 \mathrm{~b}$ \\
\hline
\end{tabular}




\section{Discussão dos resultados}

O estudo analisa as percepções dos residentes de Pelotas - RS, Brasil, à Feira Nacional do Doce - FENADOCE - e a avaliação da imagem de marca global do festival envolvendo a relação organização do evento - impactos (positivo e negativos) percebidos - apropriação do evento - apropriação da marca/mascote. O estudo constatou que o processo está alicerçado na organização do evento e nas externalidades que ela provoca, especialmente nos custos e nos benefícios sociais que produz. $O$ valor percebido pelos residentes tem origem na (melhor ou pior) organização do evento, que produz externalidades desejáveis e indesejáveis, e impulsiona diferentes sentimentos, produzindo dois grandes construtos de saída: (1) o senso de orgulho/pertencimento ao festival, enquanto produto turístico, e (2) o valor da formiga estilizada, para os residentes.

O resultado confirma que a relação de afeto que os residentes devotam ao festival - 0 senso de pertencimento - é significativamente impulsionado conforme a avaliação que fazem da organização e dos benefícios socioculturais atribuídos ao evento. Tal resultado alinha-se com pressuposto por Small (2007) e, também, somente se confirmou o valor experiencial identificado por Chiang et al. (2017). Mas ao contrário do que fora hipotetizado, o estudo não encontrou evidências significativas de que os benefícios socioeconômicos (elementos objetivos) também afetem a construção do pertencimento, diferentemente do estudo de Chiang et al. (2017). Tal atributo implica a importância de se considerar o senso de pertencimento dos moradores, que se vinculam ao evento por identificação e consequentemente terão, conforme estudos anteriores, atitudes, comportamentos, comprometimento e até intenções de compra favoráveis (Berrozpe et al., 2019; Palmer et al., 2013; Wang et al., 2014; Choo et al., 2011).

O resultado confirma a existência de duas dimensões de análise na figura da mascote, uma dimensão simbólico-afetiva e outra econômico-utilitária, e de dois grupos bem definidos de benefícios, os benefícios socioculturais e os benefícios socioeconômicos. Tanto as facetas da mascote, quanto os dois grupos de benefícios, são correlacionados entre si, mas diferem do ponto de vista conceitual, nas relações que produzem com outros construtos. Como dito no parágrafo anterior, os benefícios socioculturais são fundamentais para o fortalecimento dos laços afetivos dos residentes com o evento propriamente dito, o que corrobora os achados de Chiang et al. (2017) e Small (2007), porém não ajudam a criar valor simbólico para a mascote, contrariando o que era esperado.

Os benefícios socioeconômicos, ao contrário do esperado, não impulsionam o sentido de pertencimento ao evento, nem agregam valor econômico ou simbólico à mascote, na visão dos residentes. Ou seja: os residentes estabelecem laços afetivos com o evento em função dos benefícios socioculturais que esta lhes proporciona, mas não pelas oportunidades econômicas que ela cria no município, diferentemente do estipulado por Uysal et al. (2016). Os benefícios socioeconômicos relacionam-se fortemente à [boa] organização do evento, e são vistos como algo utilitário, como uma oportunidade de emprego, de distração, ou mesmo de consumo cultural.

Ao contrário do que era esperado, a relação de afeto dos residentes com o evento não se transfere automaticamente à sua mascote. $O$ resultado não identifica qualquer influência 
significativa do sentimento de pertença ao evento, no valor da formiga estilizada, nem no valor econômico, nem no valor simbólico. Em outras palavras, o resultado sugere que as relações dos residentes com o evento e com a sua mascote-símbolo brotam de recompensas distintas e percorrem caminhos também distintos. O vínculo com o evento propriamente dito é afetivo, e deriva basicamente das recompensas psicológicas que os residentes identificam nas ações da organização e nos benefícios socioculturais do evento. E o vínculo com a mascote, ao contrário, é uma relação funcional-utilitária de acordo com Hem e Iversen (2004), corroborando em parte com Keller e Machado (2006), que resulta da avaliação objetiva da organização do evento e da sua capacidade de mitigar os impactos negativos do mesmo. Portanto, confirma-se que o entretenimento comunitário fortalece a coesão e a identidade social (Akhoondnejad, 2016; Gursoy et al., 2004; Prayag et al., 2013; Small, 2007), mas não se confirma o pressuposto de que os benefícios econômicos do evento, como as oportunidades de emprego, por exemplo, também atuariam no mesmo sentido.

\section{Considerações finais}

O trabalho oferece contribuições teóricas e gerenciais nas áreas de marketing de eventos e de gestão de marcas de turismo ao ampliar o relacionamento da imagem global da marca. Os atributos de desempenho, funcionais e tangíveis, simbólicos/emocionais e experienciais da formação da marca (Keller, 1998; Keller \& Machado, 2006) e imagem (Rola et al. 2018; Lai, 2016) foram testados pelo modelo aqui apresentado. O modelo foi alicerçado na qualidade da organização do evento e nas externalidades que ela provoca, como as percepções dos custos e nos benefícios sociais que produz, assim como o senso de pertencimento e o valor da formiga estilizada, testados sob a visão dos residentes que visitaram a Fenadoce.

Os gestores necessitam entender os efeitos psicológicos consequentes da participação dos residentes nos eventos e festivais pois estes geram efeitos subsequentes como fidelidade a marca, tendência à compra, intensão de compra, compromisso com a marca, boca-a-boca positivo, resistência a informações negativas, avaliação positiva do consumidor, satisfação do cliente, entre outros (Berrozpe et al., 2019). Além disso, os resultados da pesquisa buscam contribuir com um maior entendimento da influência dos elementos de marca na mente dos consumidores, uma vez que tanto eventos como destinos, despendem recursos monetários com comunicação e propaganda.

Nesta pesquisa, a mascote está diretamente ligada com a organização do evento, comprovando sua importância utilitária para os gestores. Sua representatividade traduz o relacionamento de marca por meio ao desempenho das instalações e da qualidade de serviço e estrutura oferecida aos residentes consumidores do festival. Ademais, conforme a literatura, sabe-se que os consumidores se identificam com uma marca pelo conjunto de benefícios que lhes proporciona. Por este motivo, para aqueles moradores com forte senso de pertencimento, o efeito sociopsicológico do festival é diretamente influenciado pelos fatores relacionados a valores experienciais (benefícios socioculturais) e funcionais da organização do evento experimentados por eles ao longo que vão participando das edições do festival. Estes efeitos são mais amplos no sentido de complexidade e impacto nas atitudes 
e comportamentos dos residentes, pois estes sentem-se parte de uma comunidade que se auto identifica com o festival.

Do ponto de vista teórico, o trabalho faz avançar o conhecimento no campo do marketing de eventos, ao propor um modelo capaz de avaliar a apropriação de uma marcasímbolo pelos residentes, algo que ainda é pouco explorado na literatura. E além da avaliação da marca e da mascote, o trabalho também contribui para o avanço do conhecimento em outra área ainda carente de completude: a importância da incorporação de um evento anual ao ideário coletivo da população local, e a necessidade de estender os laços afetivos da população à marca-símbolo do evento. Mais investigações podem ser feitas, como por exemplo, mensurar os elementos de marca como dimensão independente na relação das demais dimensões analisadas do festival.

Gerencialmente, o trabalho oferece interessantes insights para os gestores públicos e privados, que anualmente precisam alocar os recursos estruturais, humanos e tecnológicos de forma a entregar o máximo de valor a visitantes e residentes. Os achados são válidos tanto para a administração do evento estudado, quanto para o planejamento de eventos similares. O trabalho tem limitações quanto à generalização dos resultados, tendo em vista o tipo de amostra que foi utilizada (amostra de conveniência), e por utilizar apenas residentes que visitaram a Fenadoce durante o período de coleta. Novos trabalhos sobre o fenômeno são altamente recomendáveis para replicar o método e o resultado, se possível utilizando amostras mais abrangentes e alargando o elenco de variáveis representativas dos custos e benefícios financeiros, que aqui não puderam ser utilizadas por falta de aderência ao modelo.

\section{Limitações e propostas de investigação futura}

Quanto às limitações deste estudo, destaca-se o universo de pesquisa ao buscar mensurar o mascote-símbolo do evento. Embora o modelo de mensuração tenha se mostrado estatisticamente consistente, novos estudos são necessários para atestar a sua validade. Outra limitação encontrada foi quanto ao instrumento de pesquisa, embora construído de maneira a ter um número elevado de variáveis suficientes a responderem aos objetivos e as hipóteses levantadas, não permitiu inserir outras dimensões que comprovariam empiricamente, por exemplo, a atitude, comportamento, apoio, lealdade, intenções de revisita, pois tornaria o instrumento de pesquisa muito extenso e cansativo para os inquiridos.

Sugerimos investigações futuras avaliando quanto ao elemento de marca mascote influenciam nas demais percepções ligadas a formação da imagem do evento, fazendo o caminho inverso do apresentado nesta pesquisa. Além da mascote, outros elementos simbólicos de marca necessitam de maior atenção na formação da imagem global de eventos e destinos nas pesquisas. Por fim, sugere-se uma maior atenção a importante influência dos impactos percebidos na construção da imagem de eventos e destinos, o que caberia maiores investigações pela literatura. 


\section{Referências}

Aaker, D. A. (2001). Criando e administrando marcas de sucesso. São Paulo: Editora Futura.

Akhoondnejad, A. (2016). Tourist loyalty to a local cultural event: The case of Turkmen handicrafts festival. Tourism Management, 52, 468-477. doi: 10.1016/j.tourman.2015.06.027

Anderson, J. C. \& Gerbing, D. W. (1988). Structural equation modeling in practice: A review and recommended two-step approach. Psychological Bulletin, 103(3), 411. doi: 10.1037/00332909.103.3.411

Bagozzi, R. P. \& Dholakia, U. M. (2006). Antecedents and purchase consequences of customer participation in small group brand communities. International Journal of Research in Marketing, 23(1), 45-61. doi: 10.1016/j.ijresmar.2006.01.005

Bagozzi, R. P. \& Phillips, L. W. (1982). Representing and testing organizational theories: A holistic construal. Administrative Science Quarterly, 27(3), 459-489. doi: 10.2307/2392322

Bagozzi, R. P., Yi, Y. \& Phillips, L. W. (1991). Assessing construct validity in organizational research. Administrative Science Quarterly, 36, 421-458. doi: 10.2307/2393203

Berrozpe, A., Campo, S. \& Yagüe, M. J. (2019). Am I Ibiza? Measuring brand identification in the tourism context. Journal of Destination Marketing \& Management, 11, 240-250. doi: 10.1016/j.jdmm.2018.04.005

Berselli, C., Tricárico, L. T. \& Rossini, D. (2019). Os signos e símbolos do patrimônio nas ações do marketing, uma relação possível? Reflexões a partir das campanhas da Feira Nacional do Doce (Fenadoce) de Pelotas/RS, Brasil. Revista Brasileira de Pesquisa em Turismo, 13(1), 72-91. doi: 10.7784/rbtur.v13i1.1479

Brei, V. A. \& Liberali Neto, G. (2006). O uso da técnica de modelagem em equações estruturais na área de marketing: Um estudo comparativo entre publicações no Brasil e no exterior. Revista de Administração Contemporânea, 10(4), 131-151. doi: 10.1590/S1415-65552006000400007

Brown, T. A. (2006). Confirmatory factor analysis for applied research. New York: Guilford.

Caufield, K. (2012). Analyzing the effects of brand mascots on social media: Johnson City Power Board case study. Undergraduate Honors Theses. East Tennessee State University, USA.

Chiang, L., Xu, A., Kim, J., Tang, L., \& Manthiou, A. (2016). Investigating festivals and events as social gatherings: The application of social. Journal of Travel \& Tourism Marketing, 34(6), 779-792. doi: 10.1080/10548408.2016.1233927

Choo, H., Park, S.-Y. \& Petrick, J. F. (2011). The influence of the resident's identification with a tourism destination brand on their behavior. Journal of Hospitality Marketing \& Management, 20(2), 198216. doi: 10.1080/19368623.2011.536079

De Figueiredo, M. D. \& Cavedon, N. R. (2012). Com açúcar, com afeto? A profissionalização do fazer amador de doces artesanais de pelotas. Revista Interdisciplinar de Gestão Social, 1(3), 79-99.

de Rosa, A. S., Bocci, E. \& Dryjanska, L. (2019). Social representations of the European capitals and destination e-branding via multi-channel web communication. Journal of Destination Marketing \& Management, 11, 150-165. doi: 10.1016/j.jdmm.2017.05.004

Delamere, T. A. (2001). Development of a scale to measure resident attitudes toward the social impacts of community festivals, part II. Verification of the scale. Event Management, 7(1), 25-38. doi: $10.3727 / 152599501108751452$

Deng, C. Q., Li, M. \& Shen, H. (2013). Developing a measurement scale for event image. Journal of Hospitality \& Tourism Research, 39(2), 245-270. doi: 10.1177/1096348012471378 
Dos Santos, T., Zucco, F. D. \& Kraus, C. B. (2015). Imagem de eventos turísticos: Perspectivas do festival brasileiro da cerveja, Blumenau-SC. Turismo: Visão e Ação, 17(1), 128-149. doi: 10.14210/rtva.v17n1.p128-149

Ferreira, M. M., Cerqueira, F. V. \& Rieth, F. d. (2008). O doce pelotense como patrimônio imaterial: Diálogos entre o tradicional e a inovação. MÉTIS: História \& Cultura, 7(13), 91-113.

Fornell, C. \& Larcker, D. F. (1981). Evaluating structural equation models with unobservable variables and measurement error. Journal of Marketing Research, 18(1), 39-50. doi: $10.1177 / 002224378101800104$

Garretson, J. \& Niedrich, R. (2004). Spokes-characters: Creating character trust and positive brand attitudes. Journal of Advertising, 33(2), 25-36. doi: 10.1080/00913367.2004.10639159

Getz, D. \& Page, S. J. (2016). Progress and prospects for event tourism research. Tourism Management, 52, 593-631. doi: 10.1016/j.tourman.2015.03.007

Getz, D., Andersson, T. \& Carlsen, J. (2010). Festival management studies: Developing a framework and priorities for comparative and cross-cultural research. International Journal of Event and Festival Management, 1(1), 29-59. doi: 10.1108/17852951011029298

Grappi, S., \& Montanari, F. (2011). The role of social identification and hedonism in affecting tourist repatronizing behaviours: The case of an Italian festival. Tourism Management, 32(5), 1128-1140. doi: 10.1016/j.tourman.2010.10.001

Gu, H. \& Ryan, C. (2008). Place attachment, identity and community impacts of tourism. The case of a Beijing Hutong. Tourism Management, 29(4), 637-647. doi: 10.1016/j.tourman.2007.06.006

Guerreiro, M., Oom do Valle, P. \& Mendes, J. (2011). Allgarve events: Implications for the Algarve image. Tourism, 59(2), 183-202.

Gursoy, D., Kim, K. \& Uysal, M. (2004). Perceived impacts of festivals and special events by organizers: An extension and validation. Tourism Management, 25(2), 171-181. doi: 10.1016/S02615177(03)00092-X

Haobin Ye, B., Qiu Zhang, H., Huawen Shen, J. \& Goh, C. (2014). Does social identity affect residents' attitude toward tourism development? An evidence from the relaxation of the individual visit scheme. International Journal of Contemporary Hospitality Management, 26(6), 907-929. doi: 10.1108/IJCHM-01-2013-0041

Healey, M. (2009). O que é branding? Barcelona: Editorial Gustavo Gili.

Hem, L. E. \& Iversen, N. M. (2004). How to develop a destination brand logo: A qualitative and quantitative approach. Scandinavian Journal of Hospitality and Tourism, 4(2), 83-106. doi: $10.1080 / 15022250410003852$

Hoolwerff, D. (2014). Does your mascot match your brand's personality? An empirical study on how visual characteristics of a brand mascot can function as a tool for transmitting an archetypical brand personality (Dissertação de mestrado não publicada). University of Twente, Países Baixos.

Hu, L. T., \& Bentler, P. M. (1999). Cutoff criteria for fit indexes in covariance structure analysis: Conventional criteria versus new alternatives. Structural Equation Modeling: A Multidisciplinary Journal, 6(1), 1-55. doi: 10.1080/10705519909540118

Jackson, J. W. (2002). Intergroup attitudes as a function of different dimensions of group identification and perceived intergroup conflict. Self and identity, 1(1), 11-33. doi: $10.1080 / 152988602317232777$

Keller, K. L. (1998). Strategic brand management: Building, measuring, and managing brand equity. New Jersey: Prentice Hall.

Keller, K. L. \& Machado, M. (2006). Gestão estratégica de marcas. São Paulo: Pearson Prentice Hall. 
Keller, K. L., Apéria, T. \& Georgson, M. (2008). Strategic brand management: A European perspective. London: Pearson Education.

Kelly, D. M. \& Fairley, S. (2018). What about the event? How do tourism leveraging strategies affect smallscale events? Tourism Management, 64, 335-345. doi: 10.1016/j.tourman.2017.09.009

Kim, W., Jun, H., Walker, M. \& Dran, D. (2015). Evaluating the perceived social impacts of hosting largescale sport tourism events: Scale development and validation. Tourism Management, 48, 21-32. doi: 10.1016/j.tourman.2014.10.015

Kladou, S., Kavaratzis, M., Rigopoulou, I. \& Salonika, E. (2017). The role of brand elements in destination branding. Journal of Destination Marketing \& Management, 6(4), 426-435. doi: 10.1016/j.jdmm.2016.06.011

Lai, K. (2018). Influence of event image on destination image: The case of the 2008 Beijing Olympic Games. Journal of Destination Marketing \& Management, 7, 153-163. doi: 10.1016/j.jdmm.2016.09.007

López, M. B., Virto, N. R., Manzano, J. A. \& Miranda, J. G.-M. (2018). Residents' attitude as determinant of tourism sustainability: The case of Trujillo. Journal of Hospitality and Tourism Management, 35, 36-45. doi: 10.1016/j.jhtm.2018.02.002

Magalhães, A. (2020). Branding no varejo. São Paulo: Editora Senac.

Magalhães, M. O. (1993). Opulência e cultura na Província de São Pedro do Rio Grande do Sul: Um estudo sobre a história de Pelotas (1860-1890) (Dissertação de mestrado) Universidade Federal de Santa Catarina, Brasil.

Nunkoo, R. \& Gursoy, D. (2012). Residents' support for tourism: An identity perspective. Annals of Tourism Research, 39(1), 243-268. doi: 10.1016/j.annals.2011.05.006

Palmer, A., Koenig-Lewis, N. \& Jones, L. M. (2013). The effects of residents' social identity and involvement on their advocacy of incoming tourism. Tourism Management, 38, 142-151. doi: 10.1016/j.tourman.2013.02.019

Pimentel, E., Pinho, T. \& Vieira, A. (2006). Imagem da marca de um destino turístico. Turismo: Visão e Ação, 8(2), 283-299. doi: 10.14210/rtva.v8i2

Prayag, G., Hosany, S., Nunkoo, R. \& Alders, T. (2013). London residents' support for the 2012 Olympic Games: The mediating effect of overall attitude. Tourism Management, 36, 629-640. doi: 10.1016/j.tourman.2012.08.003

$\underline{Q}$, H., Kim, L. H. \& Im, H. H. (2011). A model of destination branding: Integrating the concepts of the branding and destination image. Tourism Management, 32(3), 465-476. doi: 10.1016/j.tourman.2010.03.014

Queiroz, D., de Carvalho, Í. B., Macedo, S. B. \& Gosling, M. (2019). Eventos e mindfulness. Um estudo no Festival Vibra: Arte, esporte e música. Rosa dos Ventos-Turismo e Hospitalidade, 11(1). doi: 10.18226/21789061.v11i1p117

Rola, M., Malheiro, A. \& Sousa, B. (2018). O papel da imagem dos eventos culturais na construção da marca de um destino turístico: O caso do evento "Braga Romana". Dos Algarves: $A$ Multidisciplinary e-Journal, 33, 1-21. doi: 10.18089/DAMeJ.2018.33.1

Small, K. (2007). Social dimensions of community festivals: An application of factor analysis in the development of the social impact perception (SIP) scale. Event Management, 11(1-2), 45-55. doi: $10.3727 / 152599508783943219$

Stankova, M. \& Vassenska, I. (2015). Raising cultural awareness of local traditions through festival tourism. Tourism \& Management Studies, 11(1), 120-127.

Tajfel, H. (1978). Social categorization, social identity and social comparisons. In H. Tajfel (Ed.), Differentiation between social groups: Studies in the social psychology of intergroup relations (pp. 61-76). London: Academic Press. 
Tuškej, U., Golob, U. \& Podnar, K. (2013). The role of consumer-brand identification in building brand relationships. Journal of Business Research, 66(1), 53-59. doi: 10.1016/j.jbusres.2011.07.022

Uysal, M., Sirgy, M. J., Woo, E. \& Kim, H. L. (2016). Quality of life (QOL) and well-being research in tourism. Tourism Management, 53, 244-261. doi: 10.1016/j.tourman.2015.07.013

Vásquez, R. P. (2007). Identidade de marca, gestão e comunicação. Organicom, 7, 198-211. doi: 10.11606/issn.2238-2593.organicom.2007.138952

Wang, S. \& Chen, J. S. (2015). The influence of place identity on perceived tourism impacts. Annals of Tourism Research, 52, 16-28. doi: 10.1016/j.annals.2015.02.016

Wang, S., Zhou, L., Lee, S. \& King, C. (2014). Analysis of residents' social identity, tourism engagement, and propensity for tourism advocacy. Advances in Hospitality and Leisure, 10, 109-129. doi: 10.1108/S1745-354220140000010006

Weszka, P. (2011). FIFA World cup brand elements and local inspirations. Sport Marketing Quarterly, 20(3), 174-184.

Wheeler, F., Frost, W. \& Weiler, B. (2011). Destination brand identity, values, and community: A case study from rural Victoria, Australia. Journal of Travel \& Tourism Marketing, 28(1), 13-26. doi: 10.1080/10548408.2011.535441

Zhang, H. \& Xu, H. (2019). Impact of destination psychological ownership on residents' "place citizenship behaviour". Journal of Destination Marketing \& Management, 14, 100391 . doi: 10.1016/j.jdmm.2019.100391

Cristiane Berselu é doutoranda no Programa de Pós-Graduação em Turismo e Hotelaria da Universidade do Vale do Itajaí (UNIVALI), Balneário Camboriú, SC, Brasil. Especialista em Gestão Pública e Desenvolvimento Regional; Bacharel em Turismo e Tecnóloga em Hotelaria pela Universidade Federal de Pelotas (UFPEL), Brasil. Professora no Curso Superior de Tecnologia em Hotelaria na Universidade Federal de Pelotas (UFPEL). Membro do Conselho Municipal de Turismo de Canguçu (RS). Turismóloga na Prefeitura Municipal de Canguçu (RS). Os seus interesses de investigação incluem gestão do turismo, marketing turístico, eventos, festivais e impactos do turismo. Endereço institucional: Universidade do Vale do Itajai (UNIVALI). Campus 738 Balneário Camboriú, 5. ${ }^{a}$ Avenida, 1.100 - Bloco 07, Sala 204 - Bairro dos Municípios, CEP 88337-300, 739 Balneário Camboriú (SC), Brasil.

FABRICIA DURIEUx Zucco é doutora em Administração pela Universidade Nove de Julho (UNINOVE). Mestre em Administração; Especialista em Marketing e Bacharel em Publicidade e Propaganda pela Universidade Regional de Blumenau (FURB). Professora do Programa de Pós-graduação em Turismo e Hotelaria da Universidade do vale do Itajaí (UNIVALI) e professora da graduação da Universidade Regional de Blumenau (FURB). Membro do grupo de pesquisa HGTUR/UNIVALI: Hotelaria, Gastronomia e Serviços Jurídicos. Os seus interesses de investigação são gestão do turismo, festivais, comunicação digital. Endereço institucional: Universidade do Vale do Itajai (UNIVALI). Campus Balneário Camboriú, 5. ${ }^{a}$ Avenida, 1.100 - Bloco 07, Sala 204 - Bairro dos Municípios, CEP 88337-300, Balneário Camboriú (SC), Brasil.

EdAR da Silva AÑAÑa é pós-doutor em Turismo e Hotelaria pelo PPGTH/UNIVALI e Faculdade de Economia da Universidade do Algarve (Portugal). Doutor em Administração pela Universidade Federal do Rio Grande do Sul (UFRGS), com Estágio Doutoral no Instituto de Turismo e Lazer da Universidade de Economia e administração de Viena-Áustria (Wirtschaftsuniversität Wien); Doutorado-Equivalente pela Universidade do Porto (Portugal); Mestrado em Administração pela Universidade Federal do Rio 
Grande do Sul; Professor Associado na Universidade Federal de Pelotas (UFPEL); Professor Permanente do PPGA/FURG e Professor convidado da PPGTH/UNIVALI (Brasil). Os seus interesses de investigação são Marketing Turístico, Marca de Destinos e Competitividade de Destinos. Endereço institucional: Departamento de Administração, Universidade Federal de Pelotas (UFPEL). Campus Porto, rua Gomes Carneiro, 01 - Bairro Porto, CEP 96.010-610, Pelotas (RS), Brasil.

Submetido em 12 de maio de 2020

Aceite em 27 de julho de 2020 